\title{
The key incident monitoring and management system - history and role in quality improvement
}

https://doi.org/10.1515/cclm-2017-0219

Received March 12, 2017; accepted June 29, 2017; previously published online August 3, 2017

\section{Abstract}

Background: The determination of reliable, practical Quality Indicators (QIs) from presentation of the patient with a pathology request form through to the clinician receiving the report (the Total Testing Process or TTP) is a key step in identifying areas where improvement is necessary in laboratories.

Methods: The Australasian QIs programme Key Incident Monitoring and Management System (KIMMS) began in 2008. It records incidents (process defects) and episodes (occasions at which incidents may occur) to calculate incident rates. KIMMS also uses the Failure Mode Effects Analysis (FMEA) to assign quantified risk to each incident type. The system defines risk as incident frequency multiplied by both a harm rating (on a 1-10 scale) and detection difficulty score (also a 1-10 scale).

Results: Between 2008 and 2016, laboratories participating rose from 22 to 69 . Episodes rose from 13.2 to 43.4 million; incidents rose from 114,082 to 756,432 . We attribute the rise in incident rate from $0.86 \%$ to $1.75 \%$ to increased monitoring. Haemolysis shows the highest incidence (22.6\% of total incidents) and the highest risk (26.68\% of total risk). "Sample is suspected to be from the wrong patient" has the second lowest frequency, but receives the highest harm rating (10/10) and detection difficulty score (10/10), so it is calculated to be the 8 th highest risk (2.92\%). Similarly, retracted (incorrect) reports QI has the 10th highest frequency (3.9\%) but the harm/difficulty calculation confers the second highest risk (11.17\%).

Conclusions: TTP incident rates are generally low (less than $2 \%$ of observed episodes), however, incident risks, their frequencies multiplied by both ratings of harm and discovery difficulty scores, concentrate improvement

*Corresponding author: Tony Badrick, RCPAQAP, St Leonards, Sydney NSW, Australia, Phone: +61435416022 , Fax: +61293562003 , E-mail: tony.badrick@rcpaqap.com.au

Stephanie Gay and Mark Mackay: RCPAQAP, St Leonards, Sydney NSW, Australia

Ken Sikaris: Melbourne Pathology, Collingwood, Melbourne, Victoria, Australia attention and resources on the monitored incident types most important to manage.

Keywords: Failure Mode Effects Analysis (FMEA); postanalytical error; pre-analytical error; Quality Indicators.

\section{Introduction}

Clinical laboratories have recognised the importance of pre- and post-analytical errors for some time. For example, a landmark paper from 1997 by Plebani and Carraro [1], found that the distribution of mistakes found in the stat section of the Department of Laboratory Medicine of the University-Hospital of Padova was pre-analytical 68.2\%, analytical $13.3 \%$ and post-analytical $18.5 \%$. They also found that most of the laboratory mistakes (74\%) did not affect patients' outcome (clinical states). However, in 37 patients (19.6\%), laboratory mistakes were associated with further inappropriate investigations, thus resulting in an unjustifiable increase in costs. Moreover, in 12 patients (6.4\%) laboratory mistakes were associated with inappropriate care or inappropriate modification of therapy. Over the last 20 years there has been an increasing awareness of the problem of these extra-analytical errors and various schemes have been tried and/or introduced to provide laboratories with information they can use to improve their processes, e.g. Royal College of Pathologists Australasia (RCPA), Quality Assurance Programs (QAP), Key Incident Monitoring and Management System (KIMMS), College of American Pathologists (CAP) Q-Tracks, International Federation of Clinical Chemistry and Laboratory Medicine (IFCC) Model Quality Indicators (MQIs) IFCC MQI’s [2].

Performance and outcome measures can produce multiple benefits: improvement in the quality of patient care [3], support for administrative accountability, assistance in making judgements about testing quality, aid in setting priorities, facilitation of inter-provider comparisons over time and assessment of improvement effectiveness [4]. In particular, measuring relative frequencies of process errors compared to rates measured in the same way by peer laboratories, can be used to set priorities for quality improvement interventions. When other similar laboratories have lower frequencies of process defects, e.g. shorter turnaround time (TAT), then the comparison 
suggests a focus for process improvement for laboratories with longer TATs. In a similar way a risk based approach to measuring quality indicators prioritises improvements based on patient safety concerns. For example, phlebotomy can be monitored as follows: collectors of blood samples with high frequencies of haemolysed or otherwise poorly collected blood samples can be identified based on both intra and inter laboratory comparisons and targeted education and training offered to the specific collector. The effect (or lack of effect) of interventions can be measured, while at the same time accountability for good performance is established.

Indicators of the extra-analytical phases of the Total Testing Process (TTP) have been developed in several countries, such as Australia and New Zealand [3], The US [5, 6] Brazil [7], and Spain/Catalonia [8], and other surveys and programs have been promoted in the UK $[9,10]$, China and Croatia [11]. In 2008 the IFCC launched a Working Group named "Laboratory Errors and Patient Safety" (WG:LEPS) to identify QIs and related quality specifications which (i) produce benchmarks from comparing laboratories, (ii) promote error reduction and (iii) increase patient safety. The IFCC has developed Model Quality Indicators (MQIs) which laboratories in several countries have evaluated and the WG:LEPS has reported preliminary results $[12,13]$.

This article describes the evolution of the Royal College of Pathologists Australasia (RCPA), Quality Assurance Programs (QAP), Key Incident Monitoring and Management System (KIMMS) from an initial survey of pre-analytical errors through an External Quality Assurance (EQA) programme that measured the frequencies of pre- and post-analytic incidents to an EQA that also calculates the risks of the key incidents.

\section{Key Incident Monitoring and Management System (KIMMS) development}

The Australasian programme began with a survey looking at the pre-analytical and analytical error rates in seven public and seven private hospitals. The results showed high error rates (up to 39\% for pre-analytical errors and 26\% for analytical errors [3]). In Australia, between 1998 and 2006, the issue of pre-analytical performance, now established as a real issue, was investigated by several different bodies associated with the Royal College of Pathologists of Australasia and Pacific Laboratory Medicine Services. Pathologists' focus was on incidents where a sample needed to be recollected, a requested test was unable to be reported, or a result was amended (i.e. corrected). It quickly became apparent that risk needed to be included because not all incidents carry the same risk to patient safety [3]. Laboratory Information System (LIS) software could not monitor incidents (errors, problems, complaints), so laboratorians developed a workable model (separate from the LIS) for collecting information on incidents. The model asks three basic questions of each sample received: Is the labelling/identification acceptable? Is the sample appropriate for the requested test? Is the result reported correct and received in the correct place? Table 1 lists the key incidents to be monitored and managed in the KIMMS final model.

KIMMS acts as a system for collecting data from local laboratory incidents. Reported data can then be further aggregated to assess problems across the profession. KIMMS does not assess the adequacy of local incident management for accreditation purposes. Such assessment cannot be done from aggregated data.

\section{Key incidents}

Key incidents represent steps in the process of collecting a specimen in the pre-analytical stage and the reporting results in the post-analytical stage. For the initial pilot in 2006, there were 17 QIs divided into three categories (Table 1). By 2016 the QIs had risen to 21 and were divided into four categories (Table 2A-D).

Table 1: KIMMS initial incident categories and Quality Indicators.

\begin{tabular}{ll}
\hline Sample & - Sample known to be from wrong patient \\
misidentification & - Fewer than 2 appropriate identifiers \\
& - Inconsistency between request form and \\
& sample ID \\
& - Any within-laboratory failure in ID of sample \\
& - Lack of appropriate signatures (cross match, \\
& group and hold, genetics) \\
& - Other \\
& - Sample misidentification \\
Sample & - Incorrect patient preparation \\
recollection & - Sample haemolysed \\
& - Sample clotted \\
& - Incorrect fill level of sample \\
& - Incorrect sample storage or transport \\
& - Unlabelled sample \\
& - Incorrect patient collected \\
& - Other \\
& - Result/s modified because of an error after \\
& release in any form by the laboratory \\
Result retraction & - Results released to wrong doctor \\
or correction &
\end{tabular}


Table 2: KIMMS incident categories and QI's in 2016.

\section{Q।}

Pre-analytical

A. Identification incidents - All incidents should be counted, whether they lead to rejection or not. Incidents for precious and transfusion samples should be counted separately. Note that in the context of KIMMS, a sample may be a sample or a specimen $^{\mathrm{a}}$

a
Insufficient identifiers
initially supplied $(<3$
identifiers for Australian laboratories)

Mislabelled samples

Unlabelled/mislabelled transfusion samples

Transfusion documentation

Unlabelled/mislabelled precious samples

B. Collection and transport incidents - Incidents that lead to rejection due to collection and transport should be reported here (please include storage issues that occur during this time)

Sample haemolysed

Sample clotted

Incorrect fill level of sample Insufficient sample Incorrect patient preparation, sample transport or storage during collection and transport
Definition/example

E.g. Sample and request form from Patient A, all apparently correctly labelled. However, sample is from Patient $B$, i.e. from a completely different patient. This category applies to all types of sample, i.e. urines, swabs, blood cultures, biopsies, etc. The terminology WBIT is used widely in the literature and has been included here. Excludes any incidents in precious or transfusion samples, which are recorded separately

E.g. Any unlabelled samples for a collection event, including cases where some samples were labelled and some were not, e.g. Full Blood Count (FBC) tube OK but chemistry tube unlabelled. Excludes any incidents in precious or transfusion samples, which are recorded separately

E.g. Full name and Date of Birth (DOB) but no Medical Record Number (MRN)/ National Health Identifier (NHI)/Unit Record Number (URN) etc. Note that the minimum labelling requirements may vary from country to country. Please use whatever requirements are acceptable to your laboratory. Excludes any incidents in precious or transfusion samples, which are recorded separately

Any episode where identification on the sample does not match the information on the request, e.g. clearly different name, spelling of name different, DOB different, married vs. maiden name used, incorrect MRN or laboratory number noted on the request and or samples. Excludes any incidents in precious or transfusion samples, which are recorded separately

Unlabelled/mislabelled/mismatched / wrong blood in tube incidents for transfusion samples

In Australia and New Zealand, it is expected that all unlabelled blood bank (transfusion) samples would be rejected, in line with Australian and New Zealand Society of Blood Transfusion (ANZSBT) and National Pathology Accreditation Advisory Council (NPAAC) guidelines. This should not include samples just for group and antibody screen where these are differentiated from cross-match or group and hold samples

No collection signature/no date of collection/no consent/collection not witnessed, as defined by ANZSBT guidelines. if not able to distinguish these errors from unlabelled/mislabelled transfusion samples, or not applicable to your laboratory, report all as unlabelled/mislabelled transfusion sample

A precious/semi-precious sample is one that is not possible, difficult or too urgent to recollect, e.g. fine needle aspirate, biopsy, Cerebrospinal Fluid (CSF), amniotic fluid, or surgical samples. Each laboratory should have a definition of such circumstances that would be consistent with good practise. Our reports will refer to all these samples as precious

Any sample showing haemolysis whether accepted or rejected

E.g. Clotted FBC tubes
E.g. ESR or other whole blood/plasma tubes or syringes not filled to correct level Sample provided is not sufficient for the tests requested

The patient has not undergone the correct preparation, or the sample has not been stored or transported correctly

Examples:

- Incorrect patient preparation (not fasting, medication taken etc.)

- Samples leaked or lost in transit

- Cytogenetic specimen pre-culture stored refrigerated causing loss of cell viability

- Inappropriately adding formalin to fresh tissue specimen

- Biochemistry or fresh cytology sample stored overnight at room temperature before analysis

- Sample not frozen, incorrect transport temperature

- Sample left and not centrifuged on time

- Delayed transportation resulting in the sample too old to process requested test 
Table 2 (continued)

\begin{tabular}{|c|c|c|}
\hline & QI & Definition/example \\
\hline \multirow{6}{*}{$\begin{array}{l}\text { C. Laboratory Incidents } \\
\text { - Incidents that lead } \\
\text { to rejection due to } \\
\text { incidents that occur } \\
\text { once the samples are } \\
\text { under the control of } \\
\text { the laboratory (which } \\
\text { includes specimen } \\
\text { reception and request } \\
\text { registration) }\end{array}$} & Sample not collected & $\begin{array}{l}\text { No appropriate sample for requested test, e.g. no collection, separate specimen } \\
\text { required, etc }\end{array}$ \\
\hline & Incorrect sample type & $\begin{array}{l}\text { The sample has been collected in the wrong tube type or the wrong type of sample } \\
\text { has been collected, e.g. PT test collected in an EDTA tube (wrong preservative } \\
\text { used), or a first stream urine was collected instead of a mid-stream urine or a } \\
\text { plasma sample instead of a serum sample, spot sample instead of time sample }\end{array}$ \\
\hline & Contaminated sample & $\begin{array}{l}\text { Any sample rejected due to contamination } \\
\text { E.g. Drip arm collection; sample cross contaminated; tipping blood from EDTA tube } \\
\text { to sodium citrate tube; drug screening - deliberate or accidental; sample diluted, } \\
\text { contaminated blood cultures }\end{array}$ \\
\hline & $\begin{array}{l}\text { Any within laboratory } \\
\text { failure of Identification }\end{array}$ & $\begin{array}{l}\text { Any within laboratory identification error not related to the collection of the } \\
\text { sample. Examples include mistakenly swapping laboratory numbers on samples } \\
\text { from different patients, aliquoting samples into a wrongly labelled tube or failure } \\
\text { to label an aliquot tube }\end{array}$ \\
\hline & $\begin{array}{l}\text { Laboratory accident/ } \\
\text { incident }\end{array}$ & $\begin{array}{l}\text { Any sample rejected due a laboratory error, e.g. sample broken in the laboratory, } \\
\text { sample lost in laboratory, total volume not recorded }\end{array}$ \\
\hline & $\begin{array}{l}\text { Data entry/registration } \\
\text { incidents }\end{array}$ & $\begin{array}{l}\text { Any registration incident that is found after data entry is complete. Examples include } \\
\text { missed tests, wrongly entered tests, data entry of incorrect patient demographic } \\
\text { information (age, sex, DOB), merging of two different patient's information. It does } \\
\text { not include incorrect information due to incorrect information being received }\end{array}$ \\
\hline \multirow{5}{*}{$\begin{array}{l}\text { D. Post-analytical } \\
\text { - Incidents (after } \\
\text { results have been } \\
\text { released) }\end{array}$} & & \\
\hline & $\begin{array}{l}\text { Report retracted (amended } \\
\text { report) }\end{array}$ & $\begin{array}{l}\text { Report retracted because of an error after release in any form by the } \\
\text { laboratory. Should include any laboratory data entry errors, analytical errors } \\
\text { or interpretation errors found after report has been issued. It does not include } \\
\text { reports reissued with additional test results or further interpretation (including } \\
\text { second opinions) }\end{array}$ \\
\hline & $\begin{array}{l}\text { Results released to wrong } \\
\text { doctor }\end{array}$ & $\begin{array}{l}\text { Results released to wrong doctor or to wrong 'Copy' doctor or not sent to 'Copy' } \\
\text { doctor; may be identified through complaints or registration audit. Causes include } \\
\text { wrong doctor codes, locations, email or HL7 message addresses, or as simple as } \\
\text { placing reports into the wrong envelope for delivery }\end{array}$ \\
\hline & $\begin{array}{l}\text { Turnaround time failure for } \\
\text { haemoglobin (expressed } \\
\text { as \% of total haemoglobin } \\
\text { tests) }\end{array}$ & $\begin{array}{l}\text { This measures the proportion of haemoglobin's that fail a laboratories stated turn- } \\
\text { around time (TAT). Each laboratory should have documented TAT's, so this QI is a } \\
\text { calculation: (number of haemoglobins failing TAT/total number of haemoglobin's) } \\
\times 100 \text {. Note the TAT may be different for urgent haemoglobin's compared to routine } \\
\text { haemoglobin's }\end{array}$ \\
\hline & TAT quoted & \\
\hline
\end{tabular}

a Specimen and sample tend to be used interchangeably by laboratory personnel and by KIMMS. They refer to something collected from or

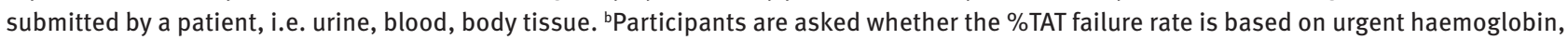
routine haemoglobin or both.

\section{Growth and problems with the development of KIMMS as an EQA system}

Under Australian accreditation, laboratories must have an incident monitoring system. The challenge for laboratories participating in KIMMS has been to align the data they already collect for accreditation to the data required by KIMMS. From 2008 until 2016, participation rose from 22 to 69 , events during which incidents could occur (episodes) from 13.2 to 43.4 million and incidents from 114,082 to 756,432 . Over this 8 year period, incidents rose from $0.86 \%$ of episodes to $1.75 \%$ of episodes; quality assurance supervisors who collected the incident data suspect that most of this doubling of the incident rate can be attributed to more complete data gathering rather than a dramatic increase in incidents. After 3 years of KIMMS we found 
that $45 \%$ of participants recorded incidents manually, a further 30\% utilise Access or Excel to capture data while a small group recorded incidents in more than one system. Laboratories often do not own or control their LIS's and are at the mercy of their IT provider for providing software modifications. Optimal configuration of current LISs to capture the KIMMS data requires support from the LIS provider, however, Australian laboratories have successfully added their own dummy test codes and configured them to record, track and report KIMMS incidents, and automated the process by using LIS equations/rules. This relies on staff to classify incidents accurately and consistently.

The data submitted for a participant is often from a pathology network which may represent the total data from many sites or laboratories. The network data reported to KIMMS are coarse and do not allow KIMMS to identify differences among each of the network laboratories; for this reason the individual laboratories lose the great benefit of external benchmarking.

In counting incidents, laboratories can attempt to count the event which caused the incident (e.g. a poor blood collection) or to count each effect of the incident (e.g. haemolysed chemistry sample for the local laboratory, missing container for full blood examination, and inadequate labelling on glycosylated haemoglobin tube for central lab). Laboratorians rarely have access to information about causative events, so they are usually wiser to count the effects of events. Similarly, just as one defective causative event can lead to several downstream defects, one downstream defect may be due to multiple upstream causes. For both these reasons, laboratorians usually count effect-events rather than cause-events.

As with many benchmarking exercises, there have been problems with the definitions and robustness of the QI's. KIMMS has tried to ensure that participants collect and submit the same data for each of the QIs. While progress has been made in this direction, the data is still not always homogeneous. For example, all laboratories assign numbers (called accessions) to incoming work in order to manage the testing process. Some laboratories have configured their LIS to allow one accession number per episode (i.e. to be used across all the different sample types/containers from one patient collection episode, even if the work spans multiple pathology departments). Other laboratory's LISs have been configured to allow a separate number to be assigned to each specimen. In KIMMS, we use episode as the denominator for incident reporting. The definition of episode is: "An episode is usually covered by a single request and may consist of one or more samples. It may be covered by more than one request, but the samples are collected at the same time: i.e. samples received together in a bag with a request form". For those laboratories that count individual sample numbers, an estimate of the number of samples per episode needs to be applied in order to meet KIMMS requirements.

\section{Harm, detection difficulty and risk}

Some incidents may impact patient safety [14]. While the absolute percentages of these harmful incidents may be small compared to the large amount of episodes, the impact of some harmful incidents on patient care may be measurable. Any potential error that impacts a patient must be managed, however, each of the QIs is not likely to have the same impact on a patient. Thus a measure of 'harm' was introduced to allow laboratories to focus on those incidents that matter most. Initially, participants were asked to decide what level of harm an incident may cause the patient: potential or none (i.e. was a quality issue). The problem with this approach was that there is no quantitation of "Harm": Harm could be as little as recollection due to a clotted tube or as much as a life threatening event as seen with a transfusion reaction due to a patient identification incident.

Another issue is that incident documenting systems do not always manage the "near miss" category of incidents well, presumably because actual incidents take priority. This meant, however, that the total risk is not well represented (A near miss event is one that could have occurred but was avoided on this occasion. It could reasonably be expected to reoccur in the future with different outcomes. Potential harm is an event that did happen, but on this occasion, there was no poor outcome for the patient). One could postulate that most incidents pathology laboratories detect are "near miss", without a consequent patient incident, which could be seen as having "potential harm" to the patient. Because "near miss" events cross-over with "potential harm" in the real world, the KIMMS programme established a single "outcome/harm" scale that makes sense of the practical daily risks laboratories face.

As well as differing in the harm that an incident may cause, QI incidents differ in the ease by which they are discovered. This variable, detection difficulty, can be scored just as harm/outcome is rated, on a scale of 1-10.

Thus in 2011, reporting the harm was changed via the use of a "risk matrix". This concept has been adapted from the Failure Mode Effects Analysis (FMEA), which estimates the risk from the (i) ability to detect an incident 
Table 3: Definitions for additional risk factors.

\begin{tabular}{ll}
\hline Additional risks & Definition/example \\
\hline $\begin{array}{l}\text { Precious samples rejected } \\
\text { Transfusion samples rejected }\end{array}$ & $\begin{array}{l}\text { The number of precious samples rejected due to identification problems } \\
\text { The number of transfusion samples rejected due to identification problems, whether these are sample or } \\
\text { documentation incidents }\end{array}$ \\
$\begin{array}{l}\text { All other unlabelled/mislabelled } \\
\text { samples rejected }\end{array}$ & $\begin{array}{l}\text { The number of samples rejected due to identification problems (not including precious and transfusion } \\
\text { samples). Note this is not all samples rejected, only those rejected due to identification incidents } \\
\text { Transfusion samples accepted } \\
\text { This is a calculation, no data required. The number of transfusion samples with identification incidents rejected } \\
\text { is subtracted from the total number of transfusion documentation and transfusion sample identification } \\
\text { incidents. It is expected that most transfusion samples are rejected, so this number should be low } \\
\text { This is a calculation, no data required. The number of precious samples and transfusion samples are } \\
\text { subtracted from the total of identification samples rejected, and the difference between Other identification } \\
\text { samples rejected and this total is the number of other identification samples accepted }\end{array}$ \\
\hline
\end{tabular}

multiplied by (ii) the frequency of the incident multiplied by (iii) the outcome or harm caused by that incident [15].

This allows each QI to be assigned a different default "risk factor" (Table 4). For identification incidents, risks are higher if samples with defective identification are accepted. These higher risks vary by sample type: more for transfusion samples, less for precious - difficult or impossible to replace - samples that are accepted despite defective identification (Table 3).

Harm and detection were defined through consensus and resulted in the KIMMS risk matrix (Table 4). It should also be noted that to date, there is no risk assigned to the TAT failure QI.

Risk in KIMMS is a calculation, the system multiplies incident frequency by harm-weight and detection difficulty from the KIMMS outcome/harm and detection difficulty estimates listed in the KIMMS outcome/harm and detection difficulty scales (Table 4). This product calculates risk as a measure of different incident types' importance and can be used for setting prevention priorities and organising counter measures. "Sample suspected to be from wrong patient" has a very low frequency $(0.4 \%$ of total incidents) but a high risk (2.92\% of total risk). In contrast, sample haemolysed has a high frequency $(22.61 \%$ of total incidents), which is reflected in its high risk $(28.68 \%$ of total risk) despite having a low harm-weight. Putting weight on the other risk multiplier, report retracted, the least frequent among the top ten incidents types (3.09\%), becomes second only to sample haemolysed as a contributor to total risk (12.01\%).

A recent international survey addressing the QIs routinely collected by laboratories shows that the "Top Ten" most adopted were (1) haemolysed samples (82.4\%); (2) sample misidentification errors (81.5\%); (3) incorrect sample type (80.9\%); (4) patient misidentification errors (78.3\%); (5) incorrect fill level (75\%); (6) clotted samples (73.6\%); (7) turnaround times outside target (70\%); (8)
Table 4: The harm and detection scores assigned to each of the KIMMS QIs.

\begin{tabular}{|c|c|c|}
\hline & \multicolumn{2}{|c|}{ KIMMS default } \\
\hline & Harm $^{\mathrm{a}}$ & Detection $^{b}$ \\
\hline \multicolumn{3}{|l|}{ Pre-analytical (before results released) } \\
\hline \multicolumn{3}{|l|}{ - Identification incidents } \\
\hline Sample suspected to be from wrong patient & 10 & 10 \\
\hline Unlabelled samples & 4 & 1 \\
\hline Minimum labelling requirements not met & 4 & 4 \\
\hline Mismatched samples & 4 & 4 \\
\hline Precious samples & 7 & 4 \\
\hline Transfusion specimen problems & 4 & 1 \\
\hline Transfusion documentation problems & 4 & 1 \\
\hline \multicolumn{3}{|l|}{ - Samples rejected/accepted } \\
\hline Precious samples with ID problems rejected & 1 & 1 \\
\hline $\begin{array}{l}\text { Transfusion samples with ID problems } \\
\text { accepted }\end{array}$ & 10 & 4 \\
\hline Other misidentified samples accepted & 7 & 1 \\
\hline \multicolumn{3}{|c|}{ - Samples rejected due to collection and transport incidents } \\
\hline Sample haemolysed & 4 & 4 \\
\hline Sample clotted & 4 & 1 \\
\hline Incorrect fill level of sample & 4 & 4 \\
\hline Insufficient sample & 4 & 1 \\
\hline $\begin{array}{l}\text { Incorrect sample collection, storage or } \\
\text { transport }\end{array}$ & 4 & 4 \\
\hline Specimen not collected & 4 & 1 \\
\hline Incorrect specimen type & 4 & 1 \\
\hline Contaminated sample & 4 & 4 \\
\hline \multicolumn{3}{|l|}{ - Samples rejected due to laboratory incidents } \\
\hline Any within laboratory failure of ID & 7 & 7 \\
\hline Test registration error & 1 & 4 \\
\hline Laboratory accident/error & 4 & 4 \\
\hline \multicolumn{3}{|l|}{ Post-analytical (after results released) } \\
\hline Report retracted & 7 & 7 \\
\hline Results released to wrong doctor & 4 & 4 \\
\hline
\end{tabular}

aHarm: 1 = potential; $4=$ recollect; $7=>$ recollect; $10=$ sentinel event (Defined by the Joint Commission (TJC) as any unanticipated event in a healthcare setting resulting in death or serious physical or psychological injury to a patient or patients, not related to the natural course of the patient's illness). 'Detection: $1=$ immediate; $4=$ probably; $7=$ unlikely; $10=$ almost impossible. 
unsuitable samples due to transportation and storage problems (67\%); (9) incorrect laboratory reports (61\%); and (10) test transcription errors (53\%) [16]. For comparison, Table 5 lists the 10 highest QI's from the KIMMS data, by frequency and by risk.

\section{Investigating the causes of incidents}

As KIMMS wanted to encourage laboratories to look at the causes of the incidents, rather than just the statistics, data are collected for two avenues of investigation. The first avenue looks at where the incident occurs, and whether it is under the supervisory control of the laboratory. The second looks at how the incident was detected: via a complaint, or through the laboratory's quality system (Table 6). As the results show, the "unknown" causes have slowly been increasing. This may well be related to an increase in participants who lacked either the capacity or interest to elucidate causes. This is a problem, as one of the KIMMS aims is to help find solutions to stop these incidents from occurring and the first step is to know who is responsible (i.e. who has authority over the situations causing the problems).

Table 5: The 10 most reported KIMMS Ql's, by frequency and by risk.

\begin{tabular}{lrr}
\hline QI & Frequency & \% Frequency \\
\hline Report retracted & 91,895 & $3.09 \%$ \\
Incorrect fill & 103,105 & $3.47 \%$ \\
Incorrect specimen & 130,118 & $4.38 \%$ \\
Mismatch or discrepant label & 142,041 & $4.78 \%$ \\
Unlabelled specimen & 174,113 & $5.86 \%$ \\
Insufficient sample & 218,616 & $7.35 \%$ \\
Sample clotted & 226,214 & $7.61 \%$ \\
Registration error & 232,549 & $7.82 \%$ \\
Specimen not collected & 537,595 & $18.08 \%$ \\
Sample haemolysed & 672,182 & $22.61 \%$ \\
& & \\
& Risk & $\%$ Risk \\
\cline { 2 - 3 } Less than 2 forms of identification & $1,095,632$ & $2.78 \%$ \\
Sample from wrong patient & $1,175,400$ & $3.13 \%$ \\
Samples rejected due to ID errors & $1,546,895$ & $4.13 \%$ \\
Incorrect fill & $1,649,680$ & $4.40 \%$ \\
Specimen not collected & $2,150,380$ & $5.74 \%$ \\
Mismatch or discrepant label & $2,272,656$ & $6.06 \%$ \\
Failure of ID within the laboratory & $3,674,118$ & $9.80 \%$ \\
Registration errors & $3,720,784$ & $9.43 \%$ \\
Report retracted & $4,502,855$ & $12.01 \%$ \\
Sample haemolysed & $10,754,912$ & $28.68 \%$ \\
\hline
\end{tabular}

a Excluding precious and transfusion samples. Data from 2011 to 2016.

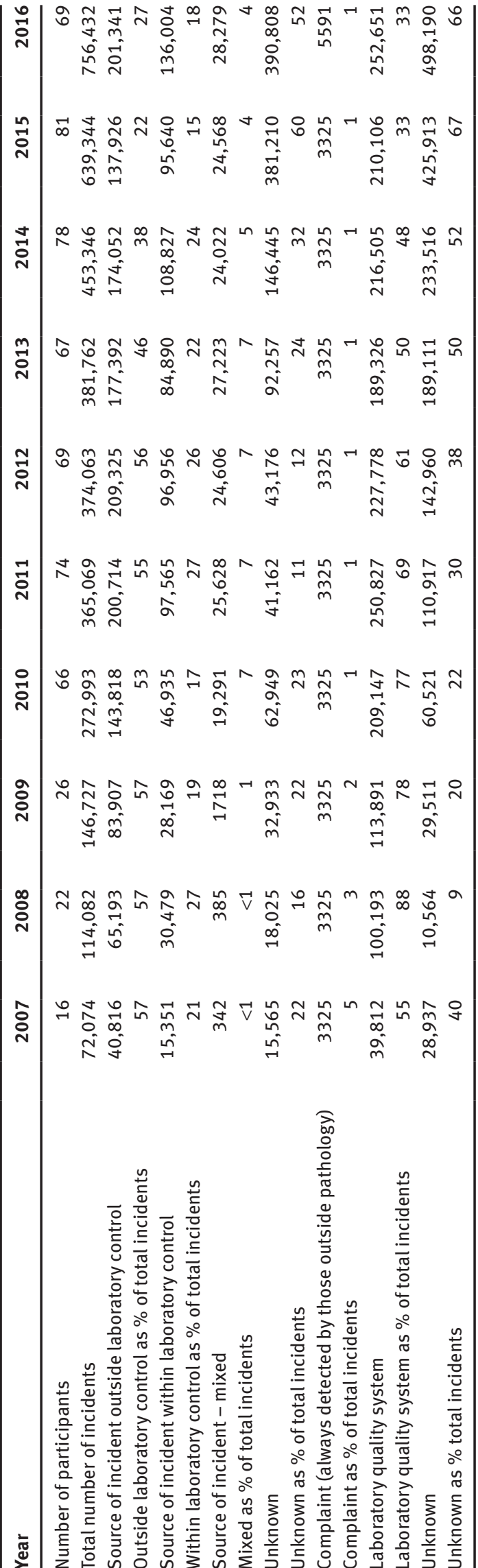




\section{Comparison to IFCC indicators}

Indicators for performance and outcome measurement allow the quality of care and services to be measured. This provides a quantitative basis for interested parties to measure improvements in care and processes by which patient care and services are provided. The measurement and monitoring of QIs in laboratory medicine serve many purposes: (i) document the quality of the service provided; (ii) improve performance and patient safety; (iii) make comparison (benchmarking) over time between laboratories; (iv) make judgments and set priorities (corrective actions to be performed); and (v) support accountability, quality improvement and accreditation.

KIMMS QIs have concentrated on pre-analytical sample integrity with some other measurements such as TAT. However, the approach taken by KIMMS is more aligned with objectives (ii), (iii) and (iv), with the addition of a risk metric and collecting information on causes and detection of key incidents.

While the IFCC WG:LEPS has presented 42 QIs, KIMMS has developed only half as many, 21. There is a strong concordance between the QIs suggested by the IFCC WG:LEPS and KIMMS for pre-analytical QIs, however, there are significant differences. KIMMS has no QI's that match the IFCC's priorities 2, 3 and 4 and makes no attempt to quantify intra-analytical QIs, as these are regularly reviewed via audits conducted by the National Association of Testing Authorities, Australia (NATA).

An overall comparison for the numbers of QIs in the IFCC WG:LEPS priority one vs. the KIMMS pre- and postanalytical stages is given in Table 7.

KIMMS has not attempted to differentiate between outpatient and inpatient QIs as this was seen to complicate an already complicated data extraction process. Rather than

Table 7: Comparison of the number of QIs for IFCC WG:LEPS vs. KIMMS.

\begin{tabular}{lrr}
\hline QI category & $\begin{array}{r}\text { Number in } \\
\text { IFCC WG:LEPS } \\
\text { category }\end{array}$ & $\begin{array}{r}\text { Number in } \\
\text { KIMMS } \\
\text { category }\end{array}$ \\
\hline Misidentification errors & 4 & 10 \\
Test(order) transcription errors & 6 & 1 \\
Sample unsuitable & 12 & 7 \\
Process delays (TAT) & 5 & 1 \\
Critical result notification failures & 2 & 0 \\
Report content errors & 1 & 1 \\
Report delay & 0 & 1 \\
Total Qls & 30 & 21 \\
\hline
\end{tabular}

aFailure to collect, wrong type, inadequate volume, defective transport or storage, specimen quality defects (contamination, haemolysis, clotting). collected separate data, KIMMS would like to benchmark data between laboratories with similar proportions of inpatient/outpatients. On the other hand, KIMMS does separate out the patient identification issues (4 IFCC WG:LEPS QIs vs. 10 KIMMS QIs) to a larger extent. This reflects the differences in risk between the types of identification issues.

More important is the difference in denominators. For all QIs KIMMS uses the total episodes (requests) as the denominator. The IFCC WG:LEPS system uses different denominator values: episodes, samples, tube types and samples submitted for testing to a specific discipline for different MQIs. The differing denominators can better highlight areas of high risk but low numbers, e.g. number of contaminated samples/microbiology specimens as compared to number of contaminated samples/total episodes. Currently, in KIMMS, the data from smaller disciplines, e.g. molecular genetics, is swamped by data from larger disciplines, e.g. chemistry and haematology.

IFCC WG:LEPS post-analytical International QIs require data on: inappropriate turn around times (time from collection to reporting, or time from arrival in the laboratory to reporting), incorrect laboratory reports, timeliness of critical result reporting, effectiveness of report comments and results notification time (time from results validation to communication to the clinician). KIMMS QIs only capture data on the number of reports retracted due to issue of wrong results, the number of results sent to the wrong doctor and TAT failure rate (see Table 7). Post-analytical data is difficult for KIMMS participants to capture and the participation in the post analytical QIs is less. Ninety-two percent of KIMMS participants report pre-analytical QIs but only 69\% of KIMMS participants report post-analytical QIs.

The missing QI for reporting critical results in KIMMS is a notable and important difference. The issue for KIMMs is the difficulty for participants to easily collect this data, however, the failure or untimeliness of receiving critical results is a high risk to the patient. Information on the timeliness of critical results reporting, the effectiveness of report comments and the results notification time, along with the current QIs of issuing wrong results and the number of results sent to the wrong doctor are probably best collected as part of an audit, that could be repeated every few years. This is currently under investigation by KIMMS. An audit would involve a more in depth view but over a limited time (i.e. one $24 \mathrm{~h}$ period).

\section{Conclusions}

The American National Academy of Medicine (formerly Institute of Medicine) Report "Improving Diagnosis in 
Healthcare" [17] highlights the importance of Diagnostic Error. It proposed in Recommendation 4A that: Health Care Organisations have programs in place to monitor the diagnostic process and identify, learn from, and reduce errors and near misses in a timely fashion. Laboratories need to set up workplace systems to detect and trap individual problems, but are also required to have a system to deal with incidents and complaints. KIMMS, as run by the RCPAQAP, is helping pathology laboratories set up detection systems, based on the most important incidents and to develop ways to investigate why and how a process fails.

To date, KIMMS has data from over 200 million episodes, and a total incident count for the QIs measured of 2.9 million. The overall incident rate stood at $1.75 \%$ for 2016. The rise in the incident rate from $0.86 \%$ in 2008 is believed to be due to participants introducing better processes to collect the data, which in itself, is a positive outcome.

Up until now, KIMMS has concentrated largely on the pre-analytical phase of the TTP cycle. Although we have three indicators for the post-analytical phase, the data is not collected well and the participation rate is low compared to other QI's (average of 91\% for pre-analytical QIs, and 69\% for post analytical QIs). Participating laboratories need to be encouraged to elucidate the causes of incidents (currently $2 / 3$ of incidents have no cause attributed to them). Both of these areas may benefit from a different type of EQA, e.g. audit.

The utility of calculating risk by multiplying the incidents frequencies by the harm-weight and detection difficulty scores assigned to incident type has not yet been fully explored. Is has been suggested that the harm-weights should be aligned with other accepted risks in the health industry in order to better educate people in positions of authority. It is acknowledged by the industry that many of the process changes required to make improvements in the areas of pre- and post-analytical testing requires the input from areas outside pathology.

KIMMS has shown that is possible to develop an EQA programme to measure pre- and post-analytical incidents, however, it requires participants to be involved in the design. If benchmarking is the goal, the incidents need to be very well defined. KIMMS needs to go beyond this goal, however, by supplying data that can be used to drive change. This is the next step.

Author contributions: All the authors have accepted responsibility for the entire content of this submitted manuscript and approved submission.

Research funding: None declared. Employment or leadership: None declared. Honorarium: None declared.
Competing interests: The funding organisation(s) played no role in the study design; in the collection, analysis, and interpretation of data; in the writing of the report; or in the decision to submit the report for publication.

\section{References}

1. Plebani M, Carraro P. Mistakes in a stat laboratory: types and frequency. Clin Chem 1997;43:1348-51.

2. Kristensen GB, Aakre KM, Sandberg S. How to conduct External Quality Assessment Schemes for the pre-analytical phase? Biochem Med 2014;24:114-22.

3. Khoury M, Burnett L, Mackay M. Error rate in Australian chemical pathology laboratories. Med J Aust 1996;165:128-30.

4. Mainz J. Defining and classifying clinical indicators for quality improvement. Int J Qual Health Care 2003;15:523-30.

5. Naklhleh RE, Souers RJ, Bashleben CP, Talbert ML, Karcher DS, Meier FA, et al. Fiftenn years' experience of a College of American Pathologists program for continuous quality improvement. Arch Pathol Lab Med 2014;138:1150-5.

6. Meier FA, Souers RJ, Howanitz PJ, Tworek JA, Perrotta PL, Nakhleh RE, et al. Seven Q-Tracks monitors of laboratory quality drive general performance improvement: experience from the College of American Pathologists Q-Tracks program 1999-2011. Arch Pathol Lab Med 2015;139:762-75.

7. Shcolnik W, de Oliveira CA, de São José AS, de Oliveira Galoro CA, Plebani M, Burnett D. Brazilian laboratory indicators program. Clin Chem Lab Med 2012;50:1923-34.

8. Kirchner MJ, Funes VA, Adzet CB, Clar MV, Escuer MI, Girona JM, et al. Quality indicators and specifications for key processes in clinical laboratories: a preliminary experience. Clin Chem Lab Med 2007;45:672-7.

9. Barth JH. Selecting clinical quality indicators for laboratory medicine. Ann Clin Biochem 2012;49:257-61.

10. Barth JH. Clinical quality indicators in laboratory medicine. Ann Clin Biochem 2012;49:9-16.

11. Simundic AM, Topic E. Quality indicators. Biochem Med 2008;18:311-9.

12. Plebani M, Sciacovelli L, Lippi G. Quality indicators for laboratory diagnostics: consensus is needed. Ann Clin Biochem 2011;48:479.

13. Sciacovelli L, O’Kane M, Skaik YA, Caciagli P, Pellegrini C, Da Rin G, et al. IFCC WG-LEPS. Quality indicators in laboratory medicine: from theory to practice. Preliminary data from the IFCC Working Group Project "Laboratory errors and patient safety”. Clin Chem Lab Med 2011;49:835-44.

14. Green SF. The cost of poor blood specimen quality and errors in preanalytical processes. Clin Biochem 2013;46:1175-9.

15. Institute for Health Improvement. Risk Priority Number. http://www.ihi.org/resources/Pages/Measures/ RiskPriorityNumberfromFailureModesandEffectsAnalysis.aspx. Accessed 7 April 2017.

16. Plebani M, O’Kane M, Vermeersch P, Cadamuro J, Oosterhuis W, Sciacovelli $L$, et al. The use of extra analytical phase quality indicators by clinical laboratories: the results of an international survey. Clin Chem Lab Med 2016;54:e315-7.

17. The Institute of Medicine. Improving diagnosis in healthcare. Washington: National Academies of Sciences, Engineering and Medicine, 2015. 